Radiologe 2009 · 49:809

DOI 10.1007/s00117-009-1919-7

Online publiziert: 23. August 2009

(c) Springer Medizin Verlag 2009

Redaktion

S. Delorme, Heidelberg

Folgender Originalbeitrag wurde für Sie gelesen und kommentiert

Sodickson A, Baeyens PF, Andriole KP et al (2009) Recurrent CT, cumulative radiation exposure, and associated radiation-induced cancer risks from CT of adults. Radiology 251:175-184

Nach einer aktuellen Erhebung liegt die mittlere effektive Dosis pro Einwohner und Jahr aufgrund diagnostischer Strahlenanwendungen in den USA mittlerweile bei 3,2 mSv, wovon etwa $45 \%$ auf CT-Untersuchungen zurückzuführen sind. Von daher überrascht es nicht, dass in letzter Zeit mehrere Publikationen amerikanischer Autoren erschienen sind, in denen sehr pointiert die Strahlenrisiken thematisiert werden, die für Patienten mit dieser aussagekräftigen Bildgebungsmodalität verbunden sind. Die neueste Studie zu dieser Thematik stammt von Sodickson et al. vom Brigham and Women's Hospital in Boston.

Es handelt sich um eine retrospektive Kohortenstudie, die an einem städtischen Lehrkrankenhaus inklusive angegliederter Krebsambulanz durchgeführt wurde. Für alle Patienten, die im Jahre 2007 mindestens eine CT-Untersuchung erhalten haben, wurden aus dem RIS alle Daten zu weiteren CT-Untersuchungen, die zwischen Mai 1986 und März 2008 stattgefunden hatten, ausgewertet. Zudem wurden weitere Patientendaten wie Alter bei Exposition und zugrunde liegende Diagnosen erfasst. Die CT-Untersuchungen wurden in 7 Kategorien eingeteilt und diesen wurde jeweils eine typische effektive Dosis zugeordnet. Auf der Basis aktueller Risikomodelle (BEIR VII) wurde dann das mit den CT-Untersuchungen assoziierte zusätzliche Lebenszeitrisiko, an einem strahleninduzierten Krebs

\title{
G. Brix
}

Abt. für medizinischen und beruflichen Strahlenschutz, Bundesamt für Strahlenschutz, Neuherberg

\section{CT-Diagnostik: Zuviel des Guten ist riskant}

zu erkranken („,lifetime attributable risk“, LAR), unter Berücksichtigung des Alters bei Exposition und Geschlecht berechnet. Insgesamt gingen die Daten von ca. 31.50o Patienten in die Analyse ein. Über den Studienzeitraum von 22 Jahren wurden bei diesen Patienten ca. 255.00o CTUntersuchungen (im Mittel ca. 8 CT-Untersuchungen/Patient, kumulative effektive Dosis $54 \mathrm{mSv}$, LAR o,3\%) durchgeführt. Etwa $33 \%$ der Patienten erhielten mehr als fünf, $5 \%$ sogar zwischen 22 und 132 CT-Untersuchungen. Für 7\% der Patienten betrug das zusätzliche Lebenszeitrisiko, an Krebs zu erkranken, mehr als $1 \%$. In dieser Untergruppe befanden sich zu 85\% Krebspatienten. Die anderen Patienten dieser Untergruppe litten z. T. ebenfalls unter schwerwiegenden Erkrankungen wie Morbus Crohn, chronischer Pankreatitis sowie perforierter Divertikulitis oder Appendizitis.

Die Arbeit von Sodickson et al. belegt eindrücklich, dass die kumulative Strahlenexposition durch diagnostische CT-Untersuchungen und das damit assoziierte Strahlenrisiko bei Patienten mit schwerwiegenden Erkrankungen, wie z. B. Krebs, erhebliche Werte erreichen kann. Methodische Limitationen der Studie - wie z. B. die Beschränkung der Datenerhebung auf ein Klinikum und damit auf eine nicht unbedingt repräsentative Patientenkohorte sowie unvermeidbare Unsicherheiten bei der Dosis- und Risikoschätzung - werden kritisch diskutiert. Nicht thematisiert werden dagegen zwei entscheidende Aspekte, nämlich der beträchtliche Nutzen, der mit den CT-Untersuchungen für die schwerkranken $\mathrm{Pa}$ tienten (hoffentlich) verbunden war sowie die Bewertung der Exposition in Relation zur eingeschränkten Lebenserwartung vieler Patienten infolge ihrer Erkran- kung. So ergab eine eigene Studie [1] interessanterweise, dass die Exposition von Tumorpatienten mit einer 5-Jahres-Überlebensrate von mehr als 70\% (z. B. Brustund Prostatatumoren) deutlich niedriger ist als diejenige von Patienten mit einer geringeren Überlebenserwartung (z. B. Pankreas- und Lungentumoren).

Nichtsdestotrotz unterstreichen die publizierten Daten einmal mehr die Notwendigkeit, insbesondere bei CT-Untersuchungen eine sorgfältige Nutzen-Risiko-Abwägung vorzunehmen. Diese hängt entscheidend von den individuellen Verhältnissen ab, wie der Art der (vermuteten) Erkrankung, dem Nutzen der Bildgebung für das Therapiemanagement, der Höhe der Strahlenexposition, dem Alter des Patienten sowie seiner erkrankungsspezifischen mittleren Lebenserwartung. Darüber hinaus sind bei diesem Abwägungsprozess, der so genannten rechtfertigenden Indikation nach $\$ 23 \mathrm{RöV}$, stets auch alternative Untersuchungsverfahren mit vergleichbarem gesundheitlichem Nutzen, die mit keiner oder einer geringeren Strahlenexposition verbunden sind, zu berücksichtigen.

\section{Korrespondenzadresse \\ Prof. Dr. G. Brix}

Abt. für medizinischen und beruflichen Strahlenschutz, Bundesamt für Strahlenschutz, Ingolstädter Landstr. 1, 85764 Neuherberg gbrix@bfs.de

Interessenkonflikt. Der korrespondierende Autor gibt an, dass kein Interessenkonflikt besteht.

\section{Literatur}

1. Brix G et al (2009) Radiation exposures of cancer patients from medical $x$-rays: how relevant are they for individual patients and population exposure? Eur J Radiol, in press 